6

\title{
The predictability of a lake phytoplankton community, from hours to years
}

Mridul K. Thomas ${ }^{1,2^{\star}}$, Simone Fontana ${ }^{1,3}$, Marta Reyes ${ }^{1}$, Michael Kehoe ${ }^{4}$, and Francesco

Pomati $^{1}$

*Corresponding author. Address: Centre for Ocean Life, DTU Aqua, Technical University of

Denmark, Kemitorvet, 2800 Kongens Lyngby, Denmark. Phone: +45 35883412

${ }^{1}$ Dept. of Aquatic Ecology, Eawag: Swiss Federal Institute of Aquatic Science and Technology, Überlandstrasse 133, 8600 Dübendorf, Switzerland

${ }^{2}$ Centre for Ocean Life, DTU Aqua, Technical University of Denmark, Kemitorvet, 2800 Kongens Lyngby, Denmark.

${ }^{3}$ Biodiversity and Conservation Biology Research Unit, Conservation Biology Group, Swiss

Federal Institute of Forest, Snow and Landscape Research, Zürcherstrasse 111, 8903

Birmensdorf, Switzerland

${ }^{4}$ Global Institute for Water Security and School of Environment and Sustainability, University of Saskatchewan

\section{Author email IDs:}

Mridul K. Thomas: mrit@dtu.dk

Simone Fontana: simone.fontana@wsl.ch

Marta Reyes: marta.reyes@eawag.ch

Michael Kehoe: kehoe.michael@eawag.ch

Francesco Pomati: francesco.pomati@eawag.ch

Key words: prediction, forecast, machine learning, phytoplankton, time series, environmental monitoring, cyanobacteria

Statement of authorship: MKT \& FP conceived the study. MKT, SF, MR \& FP collected the data. MKT analysed the data. MKT wrote the manuscript with substantial input from FP, SF, MK \&MR. 


\section{Abstract}

39 Forecasting anthropogenic changes to ecological communities is one of the central challenges in

40 ecology. However, nonlinear dependencies, biotic interactions and data limitations have limited

41 our ability to assess how predictable communities are. Here we used a machine learning

42 approach and environmental monitoring data (biological, physical and chemical) to assess the

43 predictability of phytoplankton cell density in one lake across an unprecedented range of time

44 scales. Communities were highly predictable over hours to months: model $\mathrm{R}^{2}$ decreased from

450.89 at 4 hours to 0.75 at 1 month, and in a long-term dataset lacking fine spatial resolution,

46 from 0.46 at 1 month to 0.32 at 10 years. When cyanobacterial and eukaryotic algal cell density

47 were examined separately, model-inferred environmental growth dependencies matched

48 laboratory studies, and suggested novel trade-offs governing their competition. High-frequency

49 monitoring and machine learning can help elucidate the mechanisms underlying ecological

50 dynamics and set prediction targets for process-based models. 


\section{Introduction}

61 Forecasting how environmental change will alter communities and ecosystems is perhaps the most important task facing ecologists today, and a tremendous challenge to our ecological understanding (Mouquet et al. 2015, Petchey et al. 2015, Houlahan et al. 2016). Nonlinear relationships (such as between temperature and most biological processes), stochasticity and sensitive dependence on initial conditions are sources of uncertainty that community ecology shares with other predictive disciplines, such as climate science. However, ecology additionally has to grapple with biotic interactions in complex food webs, evolutionary change, and a paucity of data with which to assess predictive power and refine models (Magurran et al. 2010). Therefore, with few exceptions (notably in disease ecology, e.g. Axelsen et al. 2015), we do not know how predictable ecological communities are (i.e. how strong the association between present and future system states is, a proxy for forecast ability). Quantifying this would allow us to understand the time scale over which we can provide actionable input for management and

73 legislative decision-making, recently termed the ecological forecast horizon (Petchey et al. 74 2015).

To make accurate long-term forecasts, ecology needs to develop process-based forecasts akin to those prevalent in climate science. Correlational approaches based on present conditions and abundances are likely to make inaccurate forecasts over decadal time-scales because patterns of environmental covariation will change in the future (Williams et al. 2007). Process-based models avoid this problem but are a challenge to design because of their complexity. This arises

80 from of a lack of knowledge of the functional forms (or shape) relating population/community

81 change to environmental factors, and a lack of data with which to parameterise them (Kremer et al. 2016). The scale of this challenge is highlighted by recent work showing that complex, highly nonlinear interactions between abiotic factors are a regular feature of physiological and

84 ecological processes (Zhu et al. 2016, Zimmer et al. 2016, Edwards et al. 2016, Thomas et al. 
2017). Designing process-based models using a traditional approach may require extensive

experimental work examining high-dimensional interactions. High-throughput screening

technologies are helping to address this problem. But in many cases, a traditional experimental

approach to understanding interactions (i.e. through multidimensional factorial experiments) may not be realistic given present funding and experimental constraints.

Machine learning (ML) algorithms offer us an alternative path towards the creation of these

process-based models. When presented with complex environmental datasets, ML allows us to

avoid the most important constraints inherent in traditional statistical approaches (a priori specification of functional forms, interactions and error distributions). Despite relying on underlying correlations, ML algorithms can improve substantially on traditional correlative analyses (Rivero-Calle et al. 2015, Kehoe et al. 2012, 2016). They can be used on complex datasets to assess associations (Rivero-Calle et al. 2015) and quantify predictability in the absence of the knowledge needed for a process-based model (Ewers et al. 2017). Even more importantly, they can be used to infer the functional forms and interactions needed to develop process-based models. This approach will require large datasets, but as ecology enters the 'big data' era, acquiring this is becoming feasible for many systems. As the cost of data acquisition continues to decrease, ML may prove a more efficient approach (relative to high-dimensional

102 factorial experiments) to assessing community predictability and understanding the drivers of 103 complex ecological dynamics.

Natural communities of microbes such as phytoplankton are vital parts of most biogeochemical cycles and food webs (Field et al. 1998, Falkowski et al. 1998), and so assessing their

106 predictability is especially important. Phytoplankton have generation times on the order of a day,

107 and respond extremely rapidly to environmental change: shifts on time-scales of minutes to

108 hours are sufficient to elicit physiological and ecological changes (Goldman \& Glibert 1982 , 
Demers et al. 1991, Hemme et al. 2014). Despite this sensitivity to environmental conditions, we do not know the time-scales over which phytoplankton community dynamics may be predicted. Historically, most plankton monitoring campaigns have measured the community at coarse timescales of once to twice a month (Jochimsen et al. 2012), amounting to tens of generations.

113 These efforts have helped us understand broad changes driven by eutrophication and environmental warming (Pomati et al. 2012, Jochimsen et al. 2012), helping to make the case for policies limiting further changes. However, with rare exceptions (notably Hunter-Cevera et al. 2014, 2016), plankton monitoring efforts have not captured data needed to accurately assess community predictability across time scales. High-frequency monitoring campaigns that sample communities and environmental drivers on sub-daily time-scales can partly address this (Pomati et al. 2011, Merel 2013, Pomati et al. 2013, Hunter-Cevera et al. 2014, 2016), filling in pieces of the picture that coarser long-term datasets have hinted at. They also provide us with the quantity of data needed to profitably employ ML tools.

We quantified the predictability of phytoplankton cell density over time scales ranging from 4 hours to 10 years, or approximately $10^{-1}$ to $10^{3}$ generations. Cell density, or the abundance of phytoplankton cells per unit volume, is the most important parameter characterising the phytoplankton community. It is a strong proxy for phytoplankton biomass (including in our system, Fig. S1) and for primary productivity, an important ecosystem property. We characterised predictability of cell density in Greifensee, a meso-eutrophic peri-alpine lake in

128 Switzerland. The Greifensee plankton community, chemistry and physics have been monitored 129 for $>30$ years, during which it has seen dramatic changes in biology as a result of eutrophication 130 and re-oligotrophication (Bürgi et al. 2003). We make use of two complementary datasets 131 examining the Greifensee phytoplankton community: (1) High-frequency data from monitoring 132 campaigns carried out in the summer and autumn of 2014 and 2015. Cell density was measured 133 at 6 different depths (Fig. 1) using scanning flow cytometry (SFCM), and environmental data 
134 were also collected (Table S1). (2) A long-term time series created from monthly measurements

135 of depth-integrated phytoplankton measurements (Fig. 1), as well as associated environmental

136 factors (Table S2). Although the datasets differ in methodology and size, they measure

137 substantially similar biological, physical and chemical factors (Tables S1, S2). Given the length

138 of the two datasets and their sampling frequency and location, we are able to directly compare

139 predictability in the two datasets at a time lag of 1 month.

140 In addition to community density, ecology aims to predict the dynamics of functional groups and

141 taxa. Especially because toxic cyanobacterial blooms are a major health concern (Chorus \&

142 Bartram 1999, Paerl \& Huisman 2009, Paerl et al. 2011, Merel et al. 2013), we also assessed

143 the predictability of cyanobacterial cell density over these time scales, and the drivers of

144 competitive dynamics between cyanobacteria and eukaryotic phytoplankton (Fig. 2). Eukaryotic

145 densities have remained relatively stable in Greifensee since the 1980s, while average

146 cyanobacterial densities first increased 100-fold during eutrophication and then decreased by a

147 similar amount over this time period as a result of re-oligotrophication (Fig. 2). Understanding the

148 drivers of growth and competition between these two broad phytoplankton groups can help us

149 refine process-based models of water quality, with important implications for the management of

150 aquatic ecosystem services.

\section{Methods}

\section{I. Overview}

153 Greifensee is a peri-alpine lake in Switzerland $\left(47.35^{\circ} \mathrm{N}, 8.68^{\circ} \mathrm{E}\right)$ with a documented history of

154 eutrophication and re-oligotrophication (Bürgi et al. 2003). The lake is currently meso-eutrophic,

$15532 \mathrm{~m}$ deep at its deepest point and just over $20 \mathrm{~m}$ deep at the sampling locations used for both

156 datasets. 
We use two datasets in this study: a high-frequency dataset consisting of measurements every 4 hours during the summer and autumn of 2014 and 2015 using the automated monitoring station Aquaprobe (Pomati et al. 2011), and a long-term dataset consisting of monthly measurements from March 1984 to June 2016. In both cases, important environmental data (both abiotic and biotic) was collected simultaneously near the middle of the lake, allowing similar analyses to be conducted and thereby enabling comparisons. However, the datasets differ in important ways:

i) The high-frequency dataset involved measurements by SFCM, while the long-term dataset involved microscopy measurements. Therefore, sampling effort and density assessment methods differ.

ii) The high-frequency dataset consists of measurements at six specific depths $(1.0,2.5,4.0,5.5$, 7.0 and $8.5 \mathrm{~m}$ ). Abiotic environmental data were also estimated at the same depth as the collected sample. In contrast, the long-term dataset consists of integrated phytoplankton measurements across the top $20 \mathrm{~m}$ of the lake. Abiotic measurements were not integrated, but collected at specific depths (except for light, which is a surface estimate), and so we calculated

171 the maximum and minimum value of each abiotic factor in the top $20 \mathrm{~m}$ for use as our predictors.

iii) The high-frequency dataset consists of 7161 measurements, while the long-term dataset 173 contains 383 measurements.

\section{High-frequency dataset generation}

176 (http://www.cytobuoy.com), to quantify the density of the total phytoplankton community as well

177 as its cyanobacterial and eukaryotic algal fractions (estimated densities are strongly correlated

178 with estimates from microscopy, Fig. S2). The CytoSense characterizes the scattering and

179 pigment fluorescence of individual phytoplankton cells. It measures cells and colonies across a 180 large proportion of the phytoplankton length range, between approximately $2 \mu \mathrm{m}$ and $1 \mathrm{~mm}$ in 
length. Particles that enter the system cross two coherent $15 \mathrm{~mW}$ solid-state lasers. The instrument's laser and sensor wavelengths are designed to target the fluorescence signals primarily from chlorophyll-a and phycocyanin, but also capture signals from phycoerythrin and carotenoids. We used two different instruments 2014 and 2015, with small differences in configuration. Instrument settings and data processing steps may be found in the supplementary information. hours, water was sampled automatically at each of the 6 depths (as described in Pomati et al. 2011). Water samples were pumped into a $150 \mathrm{~mL}$ sampling chamber at the surface through a tube with a 0.6-cm diameter opening. The sampling chamber was flushed with water from the sampling depth three to five times over 2 minutes before the CytoSense collected a subsample of up to $500 \mu \mathrm{L}$ for measurement. measured temperature, conductivity and irradiance at all depths. We also collected weekly depth-specific samples for dissolved nutrient (nitrate, phosphate, ammonium) concentration estimation, and integrated measurements of size-fractionated zooplankton. We also monitored meteorological factors including wind speed and rainfall, and made use of additional data provided by the Office of Waste, Water, Energy and Air (AWEL) of Canton Zürich on water inflow

200 (including flow rate, temperature and nutrient concentrations) into the lake. Detail of sampling

201 procedures, instruments used and measurement methodology may be found in the 202 supplementary information. 


\section{Long-term dataset}

206 1. Field sampling procedure: Approximately every month, water samples were collected for

207 physical, chemical and biological measurements near the centre of the lake $\left(47.3525^{\circ} \mathrm{N}\right.$,

$\left.2088.6748^{\circ} \mathrm{E}\right)$, approximately $1.5 \mathrm{~km}$ from the floating platform used for the high-frequency

209 measurements. For microscopic counts of the phytoplankton community, an integrated water

210 sample was collected over the upper $20 \mathrm{~m}$ of the water column with a Schröder sampler (Bürgi

211 et al. 2003).

2. Environmental factors: The full list of parameters is found in Table S2. To measure chemical

213 and physical parameters, water samples were collected every $2.5 \mathrm{~m}$ over the whole water

214 column, at the same location and on the same dates, and were analysed using standard

215 limnological methods (Rice et al. 2012). Integrated zooplankton samples were collected over the

216 upper $20 \mathrm{~m}$ of the water column. We also made use of a publicly available surface irradiance

217 dataset (Schulz et al. 2008, Müller et al. 2015) to estimate the monthly-averaged irradiance at

218 the water surface based on interpolated estimates from a location approximately $2 \mathrm{~km}$ from the

219 sampling location ( $\left.47.35^{\circ} \mathrm{N} 8.65^{\circ} \mathrm{E}\right)$. More details about sampling procedures, instruments used

220 and measurement methodology may be found in Bürgi et al. (2003).

221 3. Data processing: For every time point, we calculated the maximum and minimum value of

222 every depth-specific parameter (such as phosphate concentration) across the entire water

223 column, and used these for subsequent analyses. Additionally, samples were not collected on

224 the same day every month and, in rare cases, more than one sample was collected in a month.

225 We therefore aggregated measurements by rounding to the nearest month and then averaged

226 duplicate values.

\section{IV. Machine learning analyses}


1. Random forests overview: Random forests (RFs) are a robust ML tool comprising ensembles of regression trees (or classification trees) (Breiman 1999). In each regression 'tree' within the random 'forest', a randomly selected subset of the data is recursively partitioned based on the most strongly associated predictor. At each node, a random subset of the total number of predictors is considered for partitioning. The final tree 'prediction' for new data is given by the average value of the data within each branch of the tree. By aggregating predications across trees, RFs are able to reproduce arbitrarily complex shapes patterns without a priori functional form specification.

We took advantage of three features that make RFs a flexible and useful tool for examining ecological systems: permutation importance, easy quantification of partial effects of individual predictors, and out-of-bag prediction.

(i) The importance of each predictor in a RF is assessed by permuting the predictor across all trees in the forest and quantifying the resulting change in the forest's error rate. More important predictors lead to a greater increase in error when permuted.

(ii) The partial effect of any single predictor on the dependent variable can also be quantified,

243 allowing us to examine the functional form of the relationship (which may be arbitrarily nonlinear, 244 though non-bifurcating).

(iii) Out-of-bag (OOB) prediction allows us to make accurate estimates of error rate and goodness of fit (model $R^{2}$ ) via a process akin to cross validation (Breiman 1999). Each data

247 point is present in the training data of only a subset of all 'trees' that comprise the 'forest'.

248 Therefore, the value of every point may be predicted using the trees that have not been trained

249 with it. The 'OOB prediction error', or mean difference between the OOB predictions and the true 250 value of all points in the dataset (see Fig. S3, S4 for examples using our data) can be used to quantify the RF's predictive ability through a pseudo- $R^{2}$ : 


$$
R^{2}=1-\frac{M S E}{\operatorname{var}(y)}
$$

where MSE is the mean squared error of the OOB predictions when compared to the true values, and $\operatorname{var}(y)$ is the variance in the dependent variable. As in the case of a standard $\mathrm{R}^{2}, \mathrm{a}$ pseudo- $\mathrm{R}^{2}$ has an upper bound of 1 , indicating perfect model performance. However, note that unlike a standard $R^{2}$, there is no lower bound. It is possible for the pseudo- $R^{2}$ to be negative, if $M S E>\operatorname{var}(y)$. This may be interpreted as saying that the model prediction is worse than the mean value of the dependent variable in the entire dataset. In our analyses, we saw low, negative values of pseudo- $R^{2}$ in a small number of cases; we rounded these values to zero to avoid confusion, while noting this in the figure captions. variable was missing, and ii) the predictors sampling depth and sampling time. We omitted the latter in order to accurately estimate the effects of predictors that covary with depth and time on cell density. In other words, we believe that gradients in light, temperature and nutrients should characterise most of the relevant information contained within depth and time. predictability at time lags ranging from 4 hours to 1 month in the high-frequency dataset, and from 1 month to 10 years in the long-term dataset. For every time lag, we fit two models,

271 predicting log cell density using: 1) only log cell density at the specified time lag, and 2) both log

272 cell density and environmental parameters at the specified time lag. E.g. our simplest model

273 considering a time lag of four hours predicted log cell density at all time points using only log cell 274 density from the measurement four hours previously. 
4. Predictor importance: We assessed the importance of predictors at all time lags using the change in model error rate when the predictor values were permuted.

5. Partial effects of environment on growth: We quantified the model-inferred effects of environmental factors on cyanobacteria and eukaryotes. Instead of examining the effects of these predictors on log density, we instead examined how they influence the population growth rate (i.e. specific growth rate, day ${ }^{-1}$, the rate of change in density between successive time points). We did this to facilitate comparison between the partial effects in our field dataset and extensive prior laboratory findings for the same predictors. However, the functional forms remained highly similar to the model explaining log density.

We focussed on two factors that are known to influence phytoplankton growth (Litchman \& Klausmeier 2008) and were identified as important in our analyses: light and temperature.

Because laboratory studies typically measure the effects of environmental factors on population growth rate per day, we multiplied the estimates of growth rate over four hours by 6 to express them in the same units. We then fitted RFs to these population growth rates using environmental parameters at a 4-hour lag and estimated their partial effects. Note that we omitted log density as a predictor, but model structure was otherwise identical to those previously described.

Though we were also interested in the effects of dissolved nitrate, phosphate and N:P ratio, we had less well-resolved data for these predictors that limited the power of analyses relating to these factors.

5. Model fitting and settings: All analyses were done in the $R$ statistical environment v3.3.3 (R Core Team 2017) using the package randomforestSRC (Ishwaran \& Kogalur 2007, Ishwaran \& Kogalur 2017). We used 2000 trees for every forest, and set the number of predictors to be considered at each node to be one-third of the total number of predictors. Missing data among 
298 the predictors were imputed for the purpose of model fitting, but imputed values were not used

299 for predictor importance assessment (Ishwaran \& Kogalur 2007, Ishwaran \& Kogalur 2017).

Results

Phytoplankton cell density was highly predictable on time scales of hours to months. In our highfrequency dataset, pseudo- $R^{2}$ of the RF models trained with cell density and environmental data decreased from 0.89 at a 4 hour lag to 0.74 at a lag of 1 month (Fig. 3). The model using only cell density as a predictor had a lower $\mathrm{R}^{2}$ at all time lags. As time lag increased, including environmental data led to larger improvements in predictability: the difference in $\mathrm{R}^{2}$ between the two models was 0.03 at a 4 hour lag, and ten times higher (0.30) at a lag of 1 month (Fig. 3). In the long-term dataset, $\mathrm{R}^{2}$ of the model trained with cell density and environmental data decreased from 0.46 at a time lag of 1 month to 0.35 at 6 months, after which it remained relatively stable (Fig. 3). $\mathrm{R}^{2}$ in the density-only model was lower at all time lags.

Aside from cell density, which was the strongest predictor at all time lags in our high-frequency dataset, the most important predictors were light, temperature and thermocline depth, itself an indirect effect of temperature (Fig. 4). Light and temperature were also most important in our phosphorus and zooplankton density become more important. and long-term datasets (Fig. 3). Model $\mathrm{R}^{2}$ for cyanobacteria was consistently higher that than for

317 eukaryotes by approximately 5-20 percentage points (Fig. 3), in both types of models (cell

318 density only and cell density with environmental data).

319 To motivate the development of process-based models of phytoplankton competition, we also 320 examined the partial effects of environmental factors on the growth rate of cyanobacteria and eukaryotic algae (Fig. 5). Temperature and light, the strongest predictors in our dataset, showed 
322 biologically realistic nonlinear patterns. Additionally, in both cases, each group dominated a

323 region of parameter space, suggesting the presence of trade-offs in performance.

\section{Discussion}

325 Assessing the predictability of natural communities is crucial if we are to develop forecasts of 326 how ecosystems will be altered by anthropogenic environmental change (Petchey et al. 2015,

327 Mouquet et al. 2015, Houlahan et al. 2016). However, our ability to predict community dynamics

328 has been limited by our understanding of environmental dependencies and biotic interactions

329 (McGill et al. 2006). Our results suggest that lake phytoplankton communities are highly

330 predictable over time scales of hours to months, approximately $10^{-1}$ to $10^{2}$ generations, and

331 possibly longer (Figs. 3, S5). Our approach quantifies the decline in predictability with increasing

332 time lag, identifies the predictors that contribute to predictive power, and points towards realistic

333 trade-offs and parameterisations through the examination of partial effects. Together, these can

334 inform the development of process-based models, set targets for their forecasts to achieve, and

335 identify a forecast horizon for adaptive management strategies. This is especially true in the

336 case of cyanobacteria, which are a threat to human health and aquatic ecosystem services

337 because of toxin production, and are believed to be hard to forecast (Chorus \& Bartram 1999,

338 Paerl \& Huisman 2009, Paerl et al. 2011, Merel et al. 2013). We find cyanobacterial densities to

339 be consistently more predictable than those of eukaryotes (Fig. 3).

340 As our understanding of ecological processes improves, the limits to predictability of ecological

341 systems will be determined by more fundamental constraints such as stochasticity, and sensitive

342 dependence on initial conditions. Despite these forces, we find strong, ecologically important

343 environmental forcing in a natural system across a range of time scales (Figs. 3-5).

344 Consequently, we believe that process-based models are very likely to provide us with useful

345 predictions over medium-to-long time scales. In other words, we believe that despite the

346 complexity of phytoplankton communities, the ecological forecast horizon (Petchey et al. 2015) 
is sufficiently distant for ecologists to provide useful input into adaptive management strategies.

348 Note that we do not quantify a specific horizon here because this requires the specification of a 349 (arbitrary) forecast threshold; if desired, readers may choose these thresholds for themselves 350 and identify the resulting forecast horizon from Fig. 3.

351 Light and temperature were strongly predictive of phytoplankton dynamics across time scales 352 (Fig. 4), consistent with existing ecological understanding (Litchman \& Klausmeier 2008). We 353 also found that zooplankton density and dissolved phosphorus concentrations become highly 354 predictive on time scales longer than a year, consistent with an ongoing, multidecadal decrease 355 in phosphorus and biomass in Greifensee (Buergi et al. 2003). This identification of variables 356 that are known to play a major role in phytoplankton ecology strengthens our confidence in the 357 relationships underlying our metric of predictability. However, we note that predictor importance $358-$ while a useful tool - is sensitive to missing data patterns. Our estimates therefore understate 359 the importance of two major groups of predictors in our high frequency data: nutrients and 360 zooplankton density. Unlike most other predictors that were measured every four hours, these 361 were measured weekly in 2014 and twice a week in 2015 (Table S1). To partially correct for this 362 difference, we also assessed the relative importance of all predictors when these were 363 interpolated using generalised additive models (GAMs) (Fig. S6). Models with interpolated 364 nutrients and zooplankton predictors had marginally higher $\mathrm{R}^{2}$ values and these predictors rose 365 considerably in importance, especially dissolved nitrogen. We believe that these results are 366 noteworthy, but choose not to focus on them here because we are unable to validate the 367 interpolated estimates.

368 Importantly, the predictive power of environmental factors in our models arises from nonlinear 369 dependencies that are consistent with causal relationships established through lab studies (Fig. 370 5; Litchman \& Klausmeier 2008). Light, one of the most important predictors, has a partial effect 371 on growth that is a saturating function for cyanobacteria and a right-skewed unimodal function 
372 for eukaryotes (Fig. 5); these are the only shapes consistent with laboratory measurements of

373 light-dependent growth (Eilers \& Peeters 1998, Edwards et al. 2015). The partial effect of

374 temperature is an increasing function and possibly a left-skewed unimodal curve, consistent with

375 prior eco-physiological findings, including in phytoplankton (Kingsolver 2009, Thomas et al.

376 2012, Thomas et al. 2016). This concordance between controlled lab studies and ML-derived

377 field patterns increases our confidence in the suitability of this ML approach, and suggests that

378 the relationships we have uncovered are likely to be useful in guiding process-based model

379 creation. Furthermore, it suggests that ML approaches may be used to discover novel ecological

380 patterns. This is particularly important in the case of interactions between factors, which

381 presently require labour-intensive and expensive multifactorial experiments to understand.

The partial effects that we show here (Fig. 5) point towards trade-offs that could enable the co-

existence of cyanobacteria and eukaryotes. Cyanobacteria appear to benefit from high light

intensity and high temperature, while eukaryotes have a growth advantage in the converse

conditions. Therefore, temporal heterogeneity in one or both of these dimensions could allow for

the maintenance of both these groups (Chesson 2000). Cyanobacteria do possess higher

optimal temperatures for growth than eukaryotic phytoplankton at temperate latitudes (Thomas

et al. 2016), consistent with the temperature-dependence we see (Fig. 5). The apparent trade-off

between growth at high and low light intensities was not seen in a synthesis of lab-measured

light traits (Schwaderer et al. 2011), but at present, measurements are available only from a

small number of species and may be influenced by interactions with other factors. Laboratory

data on a broader range of species and under a greater range of conditions will be needed to resolve this discrepancy. If true, the pattern we observe in the field also suggests an explanation underlying the formation of surface scums by cyanobacteria through buoyancy regulation (Paerl et al. 2011, Carey et al. 2012). Scum formation - important due to the negative impact on lake ecosystem services - is consistent with a cyanobacterial benefit from higher irradiance. In 
degradation from surface growth. These observations offer an example of the insights that may be gained through a combination of high-frequency monitoring and machine learning.

400

401

402

403

404

405

406

407

408

409

410

411

412

413

414

415

Our models may understate the long-term predictability of the phytoplankton community. The difference in predictability between high-frequency and long-term datasets at a time lag of 1 month suggests that if a similar methodology was followed in the long-term dataset, reasonably high $\mathrm{R}^{2}$ values may have been obtained over time scales of years, not just months. This difference is driven by several factors: 1) our high-frequency dataset includes measurements of both phytoplankton and environmental factors at specific depths, as opposed to integrated values across the water column as in the long-term dataset, 2) the high-frequency dataset has more than an order of magnitude more data points (7161 vs. 383) with which to train the machine learning algorithm, and 3) the long-term dataset explores a far greater range of parameter space in temperature, nutrient concentration, zooplankton density and unmeasured factors. Of the three, we believe depth-specific sampling may be the major factor, as the difference in model $\mathrm{R}^{2}$ at a lag of one month is $<15 \%$ in the case of the cell density-only models, and $30 \%$ in the models with both cell density and environmental factors (Fig. 3). However, we also note that in more complex systems where migration is a larger factor - such as coastal and open-ocean communities - predictability may be lower unless physical circulation patterns are highly predictable as well.

It is important to note that although pseudo- $\mathrm{R}^{2}$ provides estimates of predictability that are robust (Breiman 1999), we have not assessed a true forecast, in which error is allowed to compound through time. This approach can in principle be used to make a forecast, but we chose not to do so because of large changes in environmental conditions towards the end of the 2014 and 2015 monitoring seasons. Attempting to forecast would require us to predict in conditions well outside those that the model was trained on, where it will inevitably perform poorly. Despite this limitation, we believe that out-of-bag error is a useful proxy for forecast error: the realistic 
423 environmental dependencies (Fig. 5) highlight that we are uncovering the mechanisms

424 underpinning ecological dynamics. In the future, a broader sampling of parameter space

425 (through a year-round monitoring campaign) should allow us to make and test true forecast skill.

426 We have shown that high-frequency environmental monitoring and machine learning

427 approaches can be usefully employed to uncover patterns in complex ecological communities, to

428 assess the predictability of these communities, and to uncover dependencies that can then be

429 incorporated into process-based models of communities and ecosystems. This can help us

430 address fundamental questions in ecology: What are the drivers of ecological processes and

431 how does this change through time? How large of an effect does environmental and

432 demographic stochasticity have on communities? What are the dominant trade-offs that maintain

433 diversity in natural systems and how do they operate in dynamic environments? But perhaps

434 more importantly, it can allow us to improve our forecasts of ecological systems, fulfilling a

435 fundamental obligation that ecology owes to society.

\section{Acknowledgements}

437 This work was funded by Swiss National Science Foundation grants CRSII2_147654 and 438 31003A_144053. We thank: the Office of Waste, Water, Energy and Air (AWEL) of Canton

439 Zürich for providing permission for in situ monitoring and data on water inflow into Greifensee;

440 the lab groups of H. R. Buergi and P. Spaak for data collection and access to the long-term

441 dataset; Esther Keller for assistance with microscopy; Dany Steiner, Hannele Penson and

442 Christian Ebi for help with field work and equipment maintenance; Idronaut and Cytobuoy for

443 support during monitoring campaigns; and Colin T. Kremer for helpful comments on the

444 manuscript. 


\section{References}

448 Axelsen JB, Yaari R, Grenfell BT, Stone L (2014) Multiannual forecasting of seasonal influenza

449 dynamics reveals climatic and evolutionary drivers. PNAS, 111, 9538-9542.

450 Breiman L (1999) Random Forests. Machine Learning, 45, 1-35.

451 Bürgi HR, Bührer H, Keller B (2003) Long-Term Changes in Functional Properties and

452 Biodiversity of Plankton in Lake Greifensee (Switzerland) in Response to Phosphorus

453 Reduction. Aquatic Ecosystem Health \& Management, 6, 147-158.

454 Carey CC, Ibelings BW, Hoffmann EP, Hamilton DP, Brookes JD (2012) Eco-physiological

455 adaptations that favour freshwater cyanobacteria in a changing climate. Water Research, 46,

$456 \quad 1394-1407$.

457 Chorus I, Bartram J (1999) Toxic Cyanobacteria in Water: A Guide to Their Public Health

458 Consequences, Monitoring, and Management. E \& FN Spon, 416 pp.

459 Conley DJ, Paerl HW, Howarth RW et al. (2009) Controlling eutrophication: Nitrogen and

460 Phosphorus. Science, 323, 1014-1015.

461 Demers S, Roy S, Gagnon R, Vignault C (1991) Rapid light-induced changes in cell

462 fluorescence and in xanthophyll-cycle pigments of Alexandrium excavatum (Dinophyceae) and

463 Thalassiosira pseudonana (Bacillario-phyceae): a photo-protection mechanism . Marine Ecology

464 Progress Series, 76, 185-193.

465 Edwards KF, Thomas MK, Klausmeier CA, Litchman E (2015) Light and growth in marine

466 phytoplankton: allometric, taxonomic, and environmental variation. Limnology and

467 Oceanography, 60, 540-552. 
468 Edwards KF, Thomas MK, Klausmeier CA, Litchman E (2016) Phytoplankton growth and the

469 interaction of light and temperature: A synthesis at the species and community level. Limnology

470 and Oceanography, 61, 1232-1244.

471 Eilers PHC, Peeters JCH (1988) A model for the relationship between light intensity and the rate

472 of photosynthesis in phytoplankton. Ecological Modelling, 42, 199-215.

473 Ewers RM, Andrade A, Laurance SG, Camargo JL, Lovejoy TE, Laurance WF (2017) Predicted

474 trajectories of tree community change in Amazonian rainforest fragments. Ecography, 40, 26-

47535.

476 Falkowski PG, Barber RT, Smetacek V (1998) Biogeochemical controls and feedbacks on ocean

477 primary production. Science, $\mathbf{2 8 1}, 200-206$.

478 Field CB, Behrenfeld MJ, Randerson JT, Falkowski PG (1998) Primary production of the

479 biosphere: Integrating terrestrial and oceanic components. Science, 281, 237-240.

480 Goldman JC, Glibert PM (1982) Comparative rapid ammonium uptake by four species of marine 481 phytoplankton. Limnology and Oceanography, 27, 814-827.

482 Hemme D, Veyel D, Mühlhaus T et al. (2014) Systems-Wide Analysis of Acclimation Responses

483 to Long-Term Heat Stress and Recovery in the Photosynthetic Model Organism

484 Chlamydomonas reinhardtii. The Plant Cell, 26, 4270-4297.

485 Houlahan JE, McKinney ST, Anderson TM, McGill BJ (2017) The priority of prediction in 486 ecological understanding. Oikos, 126, 1-7.

487 Hunter-Cevera KR, Neubert MG, Solow AR, Olson RJ, Shalapyonok A, Sosik HM (2014) Diel

488 size distributions reveal seasonal growth dynamics of a coastal phytoplankter. PNAS, 111, 489 9852-7. 
490 Hunter-Cevera KR, Neubert MG, Olson RJ, Solow AR, Shalapyonok A, Sosik HM (2016)

491 Physiological and ecological drivers of early spring blooms of a coastal phytoplankter. Science,

$492354,326-329$.

493 Ishwaran H. and Kogalur U.B. (2017). Random Forests for Survival, Regression and

494 Classification (RF-SRC), R package version 2.4.2.

495 Ishwaran H. and Kogalur U.B. (2007). Random survival forests for R. R News 7(2), 25-31.

496 Jochimsen MC, Kümmerlin R, Straile D (2013) Compensatory dynamics and the stability of

497 phytoplankton biomass during four decades of eutrophication and oligotrophication. Ecology

498 Letters, 16, 81-89.

499 Kehoe M, O’Brien K, Grinham A, Rissik D, Ahern KS, Maxwell P (2012) Random forest algorithm

500 yields accurate quantitative prediction models of benthic light at intertidal sites affected by toxic

501 Lyngbya majuscula blooms. Harmful Algae, 19, 46-52.

502 Kehoe MJ, Chun KP, Baulch HM (2015) Who Smells? Forecasting Taste and Odor in a Drinking

503 Water Reservoir. Environmental Science and Technology, 49, 10984-10992.

504 Kremer CT, Williams AK, Finiguerra M et al. (2016) Realizing the potential of trait-based aquatic 505 ecology: New tools and collaborative approaches. Limnology and Oceanography, 62, 253-271.

506 Litchman E, Klausmeier CA (2008) Trait-based community ecology of phytoplankton. Annual

507 Review of Ecology, Evolution, and Systematics, 39, 615-639.

508 Magurran AE, Baillie SR, Buckland ST et al. (2010) Long-term datasets in biodiversity research

509 and monitoring: assessing change in ecological communities through time. Trends in Ecology \&

510 Evolution, 25, 574-582. 
511 McGill BJ, Enquist BJ, Weiher E, Westoby M (2006) Rebuilding community ecology from

512 functional traits. Trends in Ecology \& Evolution, 21, 178-85.

513 Mouquet N, Lagadeuc Y, Devictor V et al. (2015) Predictive ecology in a changing world. Journal 514 of Applied Ecology, 52, 1293-1310.

515 Müller R, Pfeifroth U, Träger-Chatterjee C, Cremer R, Trentmann J, Hollmann R (2015) Surface

516 Solar Radiation Data Set - Heliosat (SARAH) - Edition 1.

517 Paerl HW, Hall NS, Calandrino ES (2011) Controlling harmful cyanobacterial blooms in a world

518 experiencing anthropogenic and climatic-induced change. Science of the Total Environment,

$519 \quad 409,1739-1745$.

520 Paerl HW, Huisman J (2009) Climate change: A catalyst for global expansion of harmful

521 cyanobacterial blooms. Environmental Microbiology Reports, 1, 27-37.

522 Petchey OL, Pontarp M, Massie TM et al. (2015) The ecological forecast horizon, and examples

523 of its uses and determinants. Ecology Letters, 18, 597-611.

524 Pomati F, Jokela J, Simona M, Veronesi M, Ibelings BW (2011) An automated platform for 525 phytoplankton ecology and aquatic ecosystem monitoring. Environmental Science \&

526 Technology, 45, 9658-65.

527 Pomati F, Matthews B, Jokela J, Schildknecht A, Ibelings BW (2012) Effects of re-

528 oligotrophication and climate warming on plankton richness and community stability in a deep

529 mesotrophic lake. Oikos, 121, 1317-1327.

530 Pomati F, Kraft NJB, Posch T, Eugster B, Jokela J, Ibelings BW (2013) Individual cell based

531 traits obtained by scanning flow-cytometry show selection by biotic and abiotic environmental

532 factors during a phytoplankton spring bloom. PLoS one, 8, e71677. 
533 Rice EW, Baird RB, Eaton AD, Clesceri LS (eds.) (2012) Standard Methods for the Examination

534 of Water and Wastewater, 22nd edn. American Water Works Association/American Public

535 Works Association/Water Environment Federation.

536 Rivero-Calle S, Gnanadesikan A, Del Castillo CE, Balch WM, Guikema SD (2015) Multidecadal

537 increase in North Atlantic coccolithophores and the potential role of rising CO2. Science, $\mathbf{3 5 0}$,

$538 \quad 1533-1537$.

539 Schulz J, Albert P, Behr H-D et al. (2008) Operational climate monitoring from space: the

540 EUMETSAT satellite application facility on climate monitoring (CM-SAF). Atmospheric Chemistry

541 and Physics Discussions, 8, 8517-8563.

542 Schwaderer AS, Yoshiyama K, de Tezanos Pinto P, Swenson NG, Klausmeier CA, Litchman E

543 (2011) Eco-evolutionary differences in light utilization traits and distributions of freshwater

544 phytoplankton. Limnology and Oceanography, 56, 589-598.

545 Smith VH (1983) Low nitrogen to phosphorus ratios favor dominance by blue-green algae in lake

546 phytoplankton. Science, 221, 669-671.

547 R Core Team (2017). R: A language and environment for statistical computing. R Foundation

548 for Statistical Computing, Vienna, Austria. https://www.R-project.org/.

549 Thomas MK, Kremer CT, Klausmeier CA, Litchman E (2012) A global pattern of thermal

550 adaptation in marine phytoplankton. Science, 338, 1085-1088.

551 Thomas MK, Kremer CT, Litchman E (2016) Environment and evolutionary history determine the

552 global biogeography of phytoplankton temperature traits. Global Ecology and Biogeography, 25, $553 \quad 75-86$. 
554 Thomas MK, Aranguren-Gassis M, Kremer CT, Gould MR, Anderson K, Klausmeier CA,

555 Litchman E (2017) Temperature-nutrient interactions exacerbate sensitivity to warming in

556 phytoplankton. Global Change Biology, 23, 3269-3280.

557 Williams JW, Jackson ST, Kutzbach JE (2007) Projected distributions of novel and disappearing

558 climates by 2100 AD. PNAS, 104, 5738-42.

559 Zhu K, Chiariello NR, Tobeck T, Fukami T, Field CB (2016) Nonlinear, interacting responses to

560 climate limit grassland production under global change. PNAS, 113, 10589-10594.

561 Zimmer A, Katzir I, Dekel E, Mayo AE, Alon U (2016) Prediction of multidimensional drug dose

562 responses based on measurements of drug pairs. PNAS, 113, 10442-10447. 
Fig. 1. Dynamics of cell density of the total phytoplankton community, in both the high-frequency and long-term datasets from Greifensee. High-frequency measurements were made every 4 hours in summer-fall 2014 and 2015, at six depths. Long-term measurements were made monthly from 1984 to 2016 and were integrated over the top 20m. Note that X-axes are on different scales in each panel. Y-axes are identical for the top two panels but differ for the third.
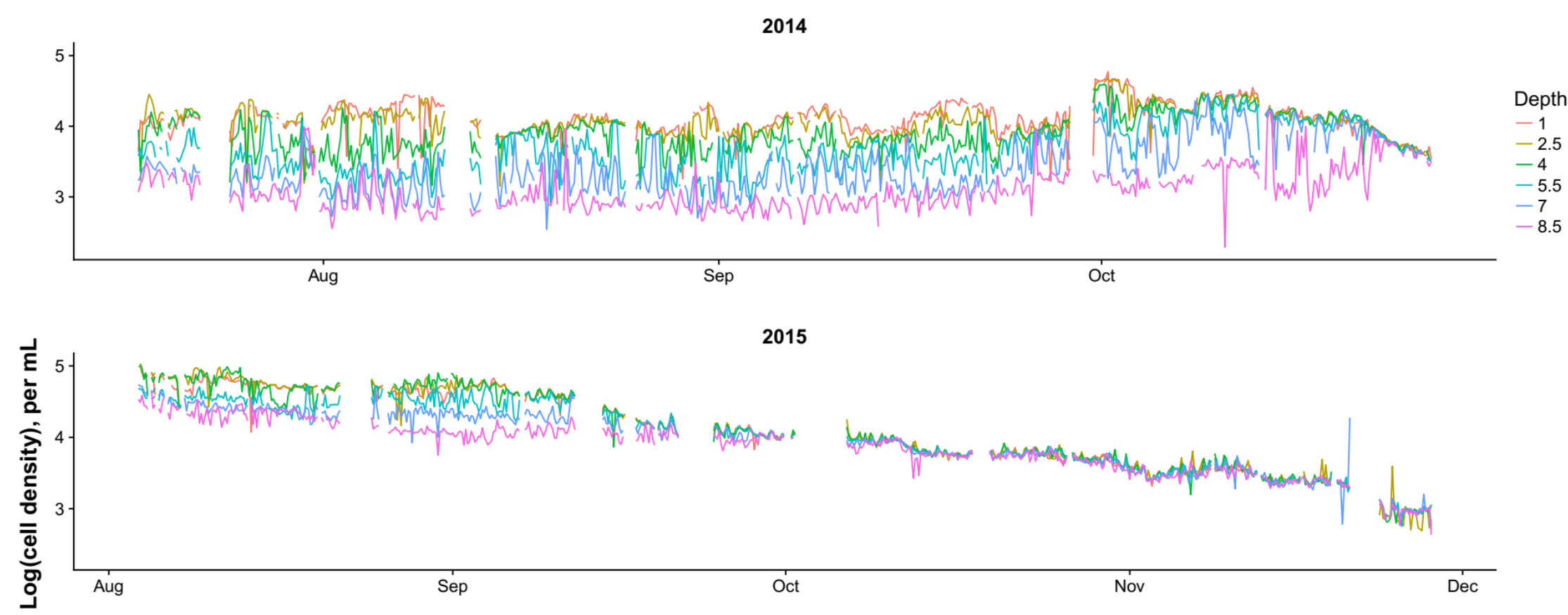

1984-2016

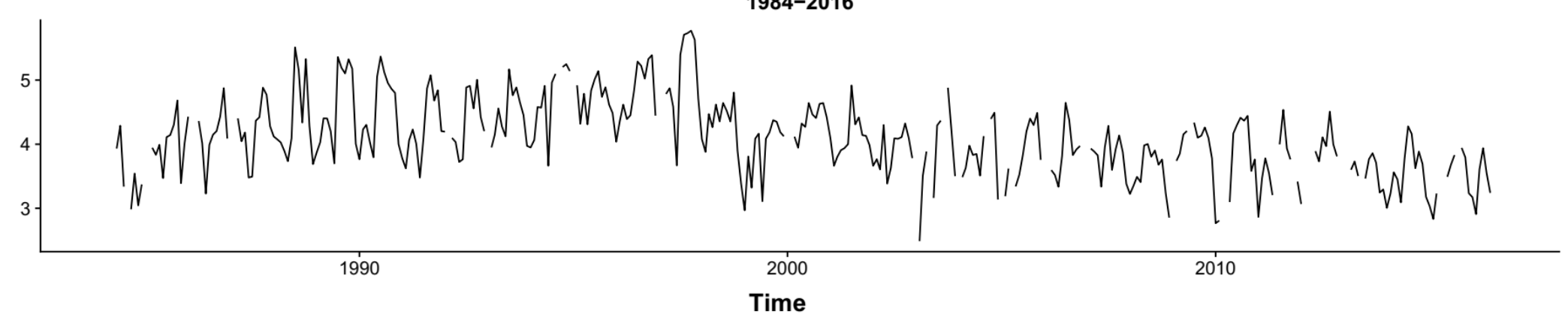


Fig. 2. Dynamics of cell density of the cyanobacteria and eukaryotic phytoplankton, in both the high-frequency and long-term datasets from Greifensee. High-frequency measurements were made every 4 hours in summer-fall 2014 and 2015 , at six depths. Long-term measurements were made monthly from 1984 to 2016 and were integrated over the top $20 \mathrm{~m}$. Note that $X$ and $\mathrm{Y}$-axes are on different scales in each column.
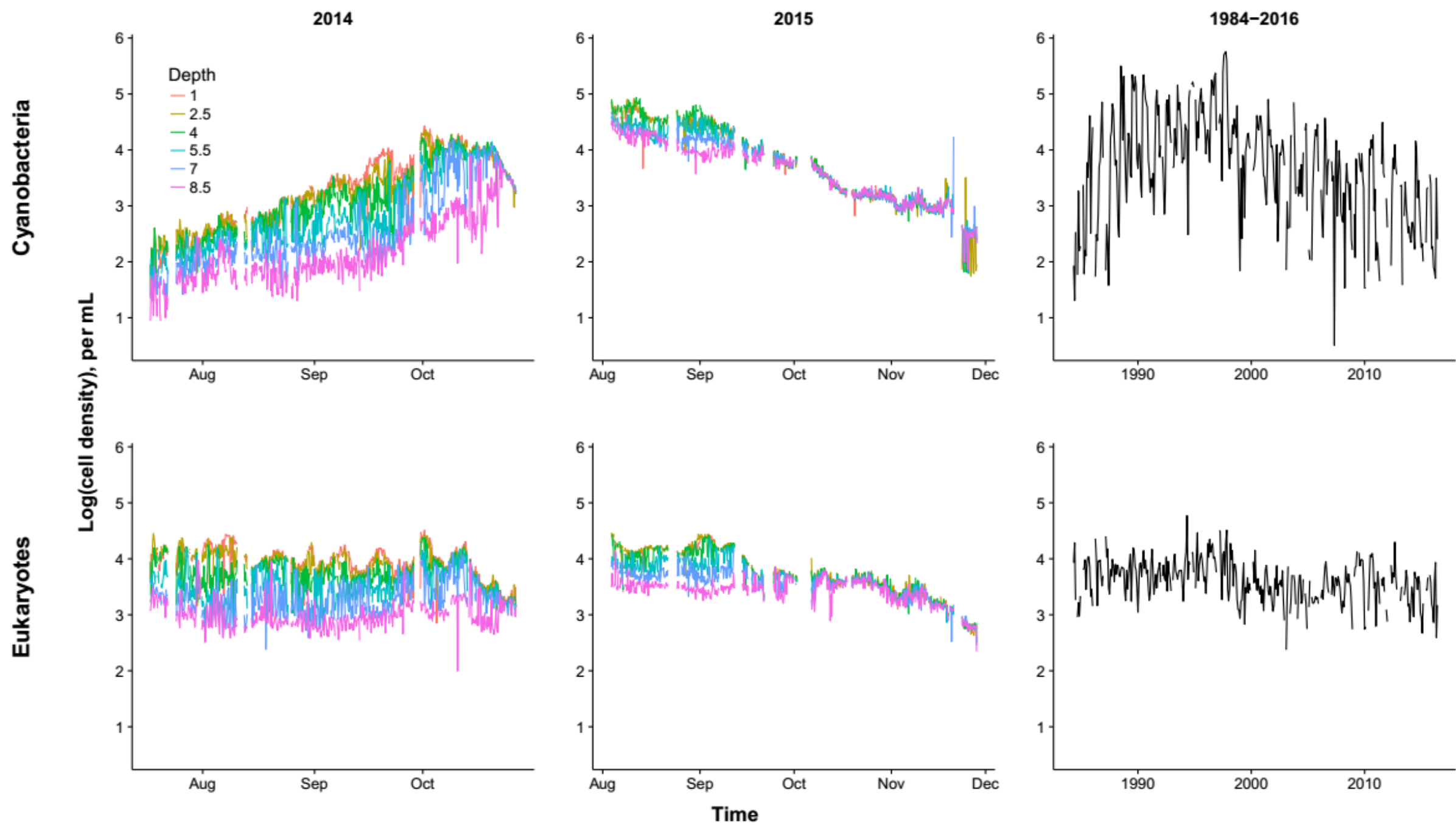
Fig. 3. Decline in predictability of the phytoplankton community with time, characterised by the random forest pseudo- $\mathbf{R}^{2}$. The predictive contribution of environmental information increased with increasing time lag (distance between solid and dashed lines increases). Cyanobacteria were consistently more predictable than eukaryotes. Despite overlap between high-frequency and long-term datasets at a time lag of 1 month, there is a decline in predictability likely driven by the lack of depth resolution in plankton and environmental data in the long-term dataset. The spike in $\mathbf{R}^{2}$ of the 'cell density only' models at 1 year reflects strong annual cycles in density. Note that pseudo- $R^{2}$ values can go negative (see Methods), and we rounded a few slightly negative values up to zero. We present the same results in terms of change in Mean Absolute Error with increasing time lag in Fig. S5.
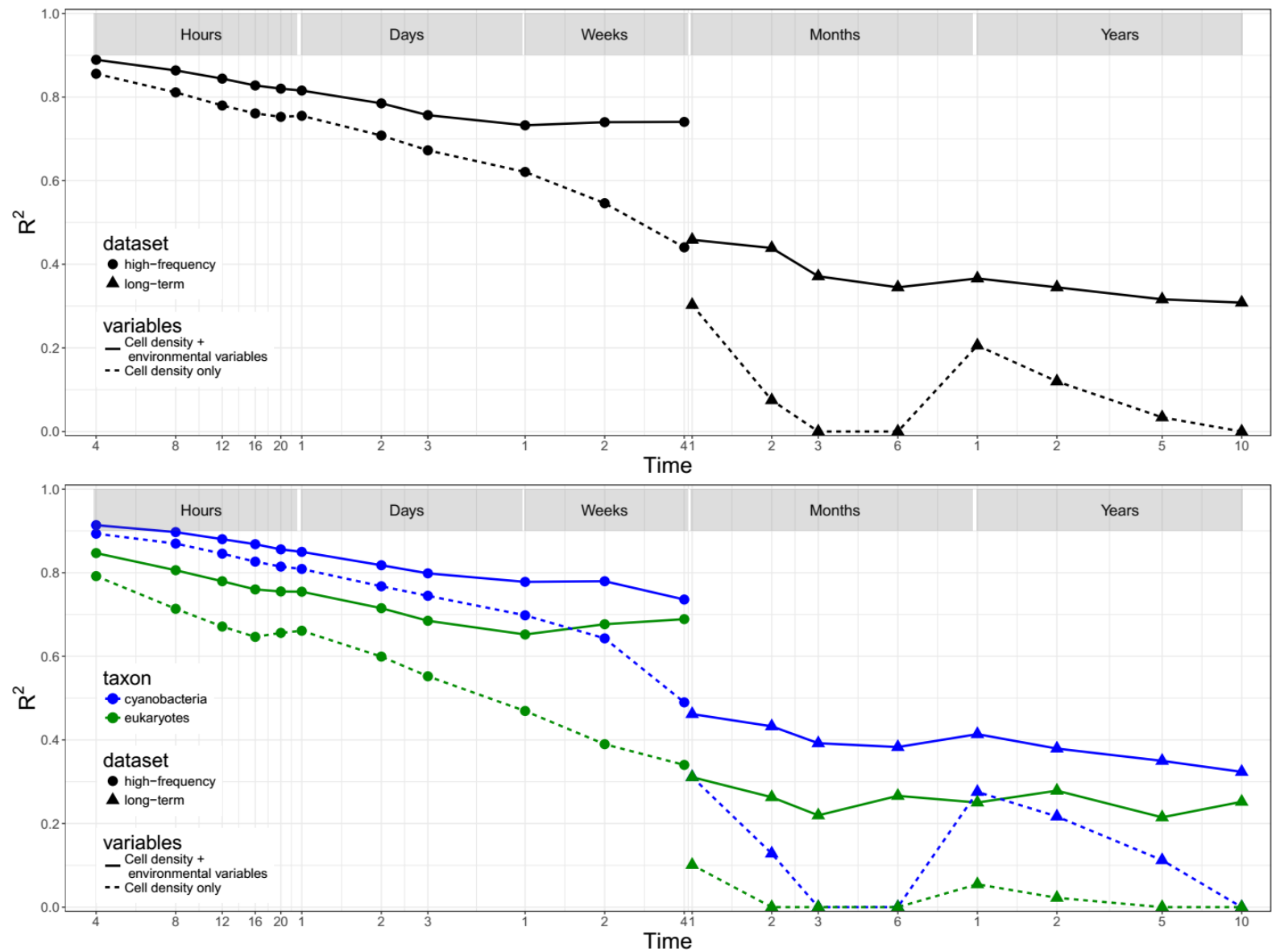
Fig. 4. The most important predictors of phytoplankton cell density at different time lags, ordered by descending rank. Light and temperature (directly, or indirectly through thermocline depth) were important predictors at most time scales. In the longterm dataset, phosphorus and zooplankton density become highly important predictors at time scales of $>1$ year. Only the most important variables are shown here, for legibility (the top 5 predictors contribute $>\mathbf{8 0} \%$ of the predictive power in most cases). In the high-frequency dataset, only variables that are in the top 10 most important for at least one time lag are shown, while in the long-term dataset we show only variables that appear in the top 5 most important at least once. See Tables S4 and S5 for the importance of all variables tested.
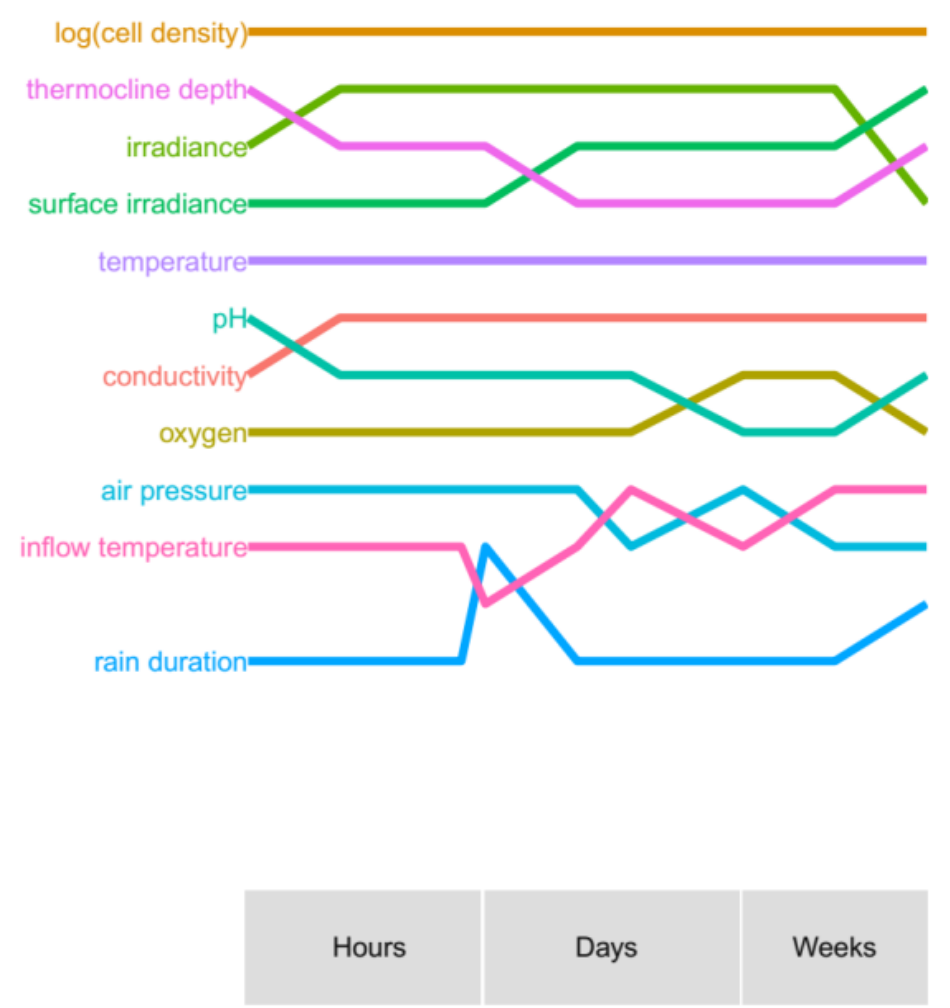

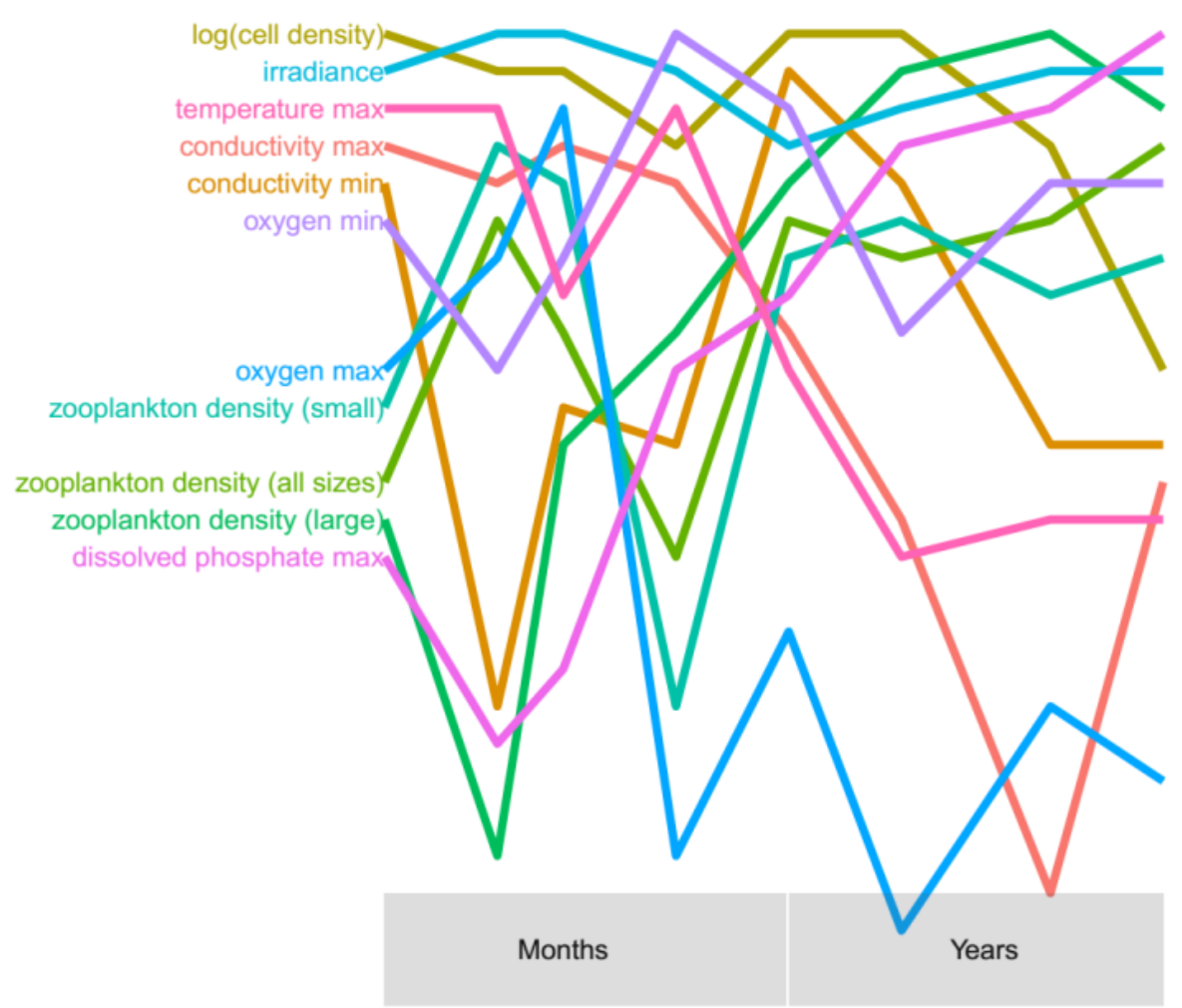


Fig. 5. Partial effects of important environmental variables on the population growth rates of cyanobacteria and eukaryotes, based on an RF model with a 4-hour time lag. The patterns reveal environmental dependencies consistent with lab experiments and suggest trade-offs with important ecological implications. Cyanobacteria appear to benefit from high light and high temperature. Wholly negative partial effects reflect the fact that in 2015, the community was decreasing through the majority of the monitoring campaign. Therefore, differences in shape and magnitude are highly informative, but the absolute estimates are only indicative. This is especially true because interactions between variables are captured by the complete forest prediction, but are not visible in single-dimension partial effects plots. Note that $Y$ axes are on different scales.

group $\bullet$ cyanobacteria $\bullet$ eukaryotes
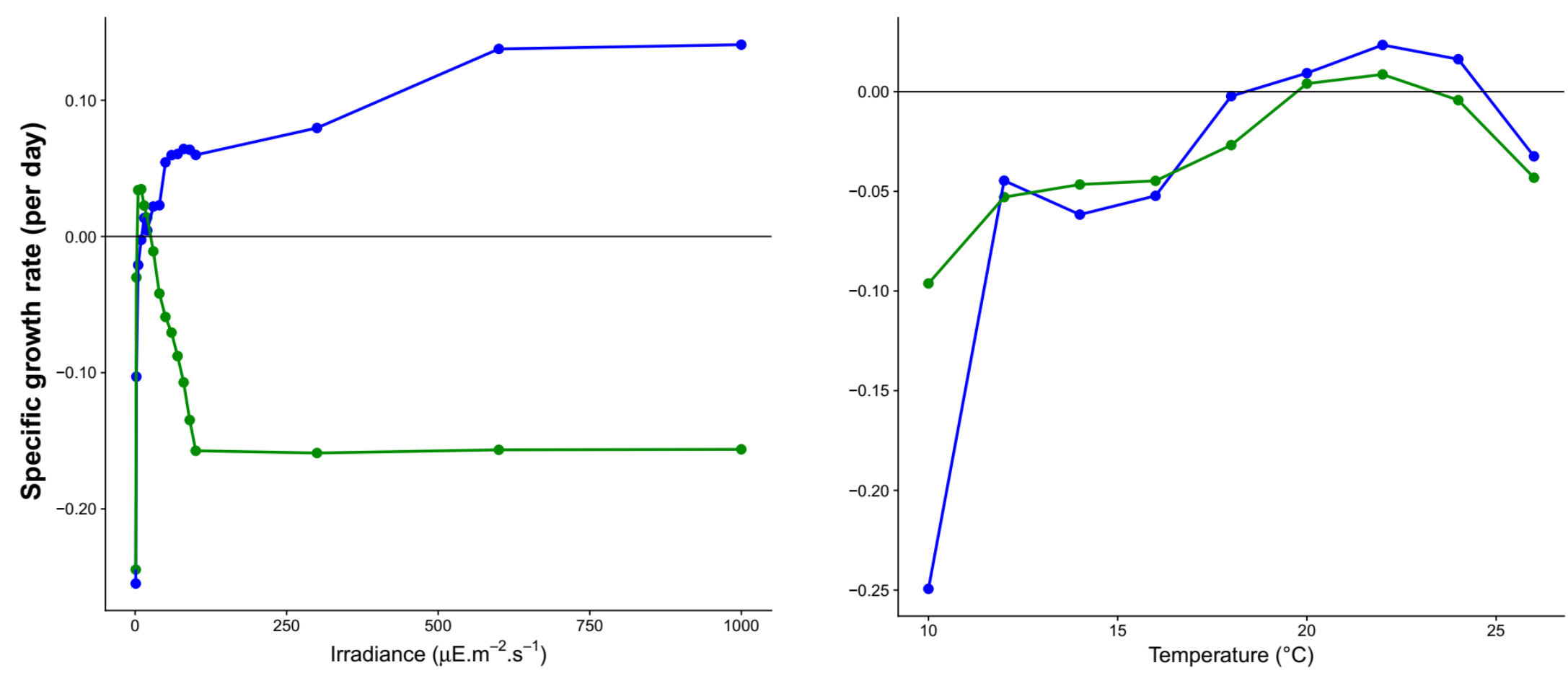